\title{
A unified view of diversity in multiantenna-multicarrier systems: analysis and adaptation strategies
}

\author{
Felip Riera-Palou* and Guillem Femenias
}

\begin{abstract}
Multicarrier and multiple transmit/receive antenna have become two key technologies underpinning most of the current development and research efforts towards ubiquitous high-throughput wireless communications. Both techniques can be used to increase the link throughput and/or to improve its robustness against channel fading and noise. This paper presents a unified bit error rate analysis for a particular flavour of multicarrier, namely, group-orthogonal code-division multiplex (GO-CDM), in combination with multiple Tx/Rx antennas. This system can be shown to encompass many of current wireless architectures and the analysis is general enough to incorporate the effects of channel frequency selectivity and Tx and/or Rx antenna correlation. The first main outcome of this paper is a general analytical framework suitable to study the effects of the different types of diversity in multicarrier systems. This analytical framework paves the way for the second main outcome of this study, namely, the design of effective reconfiguration strategies that serve to balance different system requirements (e.g., performance, complexity, delay). Particularly, it will be seen that the analytical results not only allows a-priori design decisions to be made, but it also provides an insight that enables the derivation of dynamic reconfiguration strategies that take into account instantaneous channel state information. The overall conclusion is that GO-CDM can play an important role in improving the performance of adaptive MIMO-OFDM systems.
\end{abstract}

\section{Introduction}

Most state-of-the-art wireless systems (e.g., IEEE 802.11n, IEEE $802.16 \mathrm{~m}$, 3GPP-LTE, LTE-Advanced) rely on a physical layer based on multicarrier multiantenna principles in trying to fulfill the stringent quality-of-service (QoS) requirements of modern multimedia applications. In particular, the combination of orthogonal frequency division multiplexing (OFDM) with multiple-input multipleoutput (MIMO) antenna configurations results in a powerful architecture, MIMO-OFDM, that is able to exploit the various degrees of freedom available in the wireless environment [1].

A significant improvement over conventional OFDM was the introduction of multicarrier code division multiplex (MC-CDM) by Kaiser in [2]. In MC-CDM, rather than transmitting a single symbol on each subcarrier, as

*Correspondence: felip.riera@uib.es

Mobile Communications Group, Dept. of Mathematics and Informatics, University of the Balearic Islands, 07122 Mallorca, Spain in conventional OFDM, symbols are code-division multiplexed by means of orthogonal spreading codes and simultaneously transmitted onto the available subcarriers. Since each symbol travels on more than one subcarrier, thus exploiting frequency diversity, $\mathrm{MC}-\mathrm{CDM}$ offers improved resilience against subcarrier fading. This technique resembles very much the principle behind multicarrier code-division multiple access (MC-CDMA) where each user is assigned a specific spreading code to share a group of subcarriers with other users [3]. A more flexible approach to exploit the frequency diversity of the channel is achieved by means of group-orthogonal code-division multiplex (GO-CDM) [4]. The idea behind GO-CDM, rooted in a multiple user access scheme proposed in [5], is to split suitably interleaved symbols from a given user into orthogonal groups, apply a spreading matrix on a pergroup basis and finally map each group to an orthogonal set of subcarriers. The subcarriers assigned to a group of symbols are typically chosen as separate as possible within the available bandwidth in order to maximise the

\section{是 Springer}

(c) 2012 Riera-Palou and Femenias; licensee Springer. This is an Open Access article distributed under the terms of the Creative Commons Attribution License (http://creativecommons.org/licenses/by/2.0), which permits unrestricted use, distribution, and reproduction in any medium, provided the original work is properly cited. 
frequency diversity gain. Note that a GO-CDM setup can be seen as many independent MC-CDM systems of lower dimension operating in parallel. This reduced dimension allows the use of optimum receivers for each group based on maximum likelihood (ML) detection at a reasonable computational cost.

In [4], results are given for group dimensioning and spreading code selection. In particular, it is shown that the choice of the group size should take into account the operating channel environment because an exceedingly large group size surely leads to a waste of computational resources, and even to a performance degradation if the channel is not frequency-selective enough. Modern wireless setups must be ready to deal with a large variation of possible scenarios, from small offices to large indoor/outdoor cells, and equipment configurations, from low-complexity battery-powered handsets to plugged high-end laptops. Inevitably, the conservative approach of designing the system to perform satisfactorily in the most demanding type of scenario may lead to a significant waste of computational power, thus compromising the operation of battery operated devices. In order to minimise the effects of a mismatch between the operating channel and the GO-CDM architecture, group size adaptation in the context of GO-CDM has been proposed in [6], where it is shown that important complexity reductions can be achieved by dynamically adapting the group size in connection with the sensed frequency diversity of the environment.

Complementing OFDM, multiple-antenna technology (i.e., MIMO) is the other main enabler towards high speed robust wireless networks. Expanding the traditional use of multiple antennae at the receiver side as a means to increase diversity, the application of multiple antennae at the transmitter side has been shown to lead to humongous capacity gains. In particular, the linear increase in capacity achieved when jointly increasing the number of antennas at transmission and reception, theoretically forecasted in [7], has spurred research efforts to effectively realize it in practice. To this end, three schemes have achieved notable importance in the standardisation of modern wireless communications systems, namely, spatial division multiplexing (SDM), space-time block coding (STBC) and cyclic delay diversity (CDD). While in SDM [8], independent data streams are sent from the different antennas in order to increase the transmission rate, in STBC $[9,10]$ the multiple transmission elements are used to implement a space-time code targeting the improvement of the error rate performance with respect to that achieved with single-antenna transmission. In CDD [11] a single data stream is sent from all transmitter antennae with a different cyclic delay applied to each replica, effectively resulting as if the original stream was transmitted over a channel with increased frequency diversity.
Different authors have partially addressed comparative studies between some MIMO strategies. For example, in [12] CDD and STBC are compared by means of simulation within the context of MC-CDMA, whereas [13] comparatively analyses SDM and STBC and a switching strategy between the two techniques is derived that takes into account the instantaneous channel state. However, and to the best of author's knowledge, no study has comprehensively covered the three techniques in a multicarrier context.

This paper has two main goals. The first goal is to present a unified BER analysis of the MIMO-GO-CDM architecture. In order to get an insight of the best possible performance this system can offer, attention is restricted to the case when ML detection is employed at the receiver. The analysis is general enough to incorporate the effects of channel frequency selectivity, $\mathrm{Tx} / \mathrm{Rx}$ antenna correlations and the three most common methods of spatial processing (SDM, STBC and CDD) in combination with GO-CDM frequential diversity. The analytical results are then used to explore the benefits of GO-CDM under different spatial configurations identifying the most attractive group dimensioning from a performance/complexity perspective. Based on the previous analysis and building upon our previous work for SISO OFDM systems [6], the second goal of this work is to devise effective reconfiguration strategies that can automatically and dynamically fix some of the parameters of the system, more in particular, the group size of the GO-CDM component, in response to the instantaneous channel environment with the objective of optimising some pre-defined performance criteria (e.g., error rate, complexity, delay). We note that in lieu of analytical tractability, this work solely focuses on uncoded BER performance, although most qualitative conclusions regarding what MIMO technique to use or the dimensioning of the GO-CDM component as a function of the wireless environment fundamentally hold for coded scenarios (see for example [14]).

The rest of this paper is organized as follows. Section 2 introduces the system model of a generic MIMO-GOCDM system, paying special attention to the steps required to implement the frequency spreading and the MIMO processing. In Section 3 a unified BER analysis is presented for the case of $\mathrm{ML}$ detection. In light of this analysis, Section 4 explores reconfiguration strategies aiming at the optimisation of several critical parameters of the MIMO-GO-CDM architecture. Numerical results are presented in Section 6 to validate the introduced analytical and reconfiguration procedures. Finally, the main conclusions of this work are recapped in Section 7.

Notational remark: Vectors and matrices are denoted by bold lower and upper case letters, respectively. The superscripts ${ }^{*}, T$ and ${ }^{H}$ are used to denote conjugate, transpose 
and complex transpose (Hermitian), respectively, of the corresponding variable. The operation $\operatorname{vec}(\boldsymbol{A})$ lines up the columns forming matrix $\boldsymbol{A}$ into a column vector. The symbols $\otimes$ and $\odot$ denote the Kronecker and element-byelement products of two matrices, respectively. Symbols $\boldsymbol{I}_{k}$ and $\mathbf{1}_{k \times l}$ denote the k-dimensional identity matrix and an all-ones $k \times l$ matrix, respectively. The symbol $\mathcal{D}(\boldsymbol{x})$ is used to represent a (block) diagonal matrix having $x$ at its main (block) diagonal. The determinant of a square matrix $\boldsymbol{A}$ is represented by $|\boldsymbol{A}|$ whereas $\|\boldsymbol{x}\|^{2}=\boldsymbol{x} \boldsymbol{x}^{H}$. Expression $\lceil a\rceil$ is used to denote the nearest upper integer of $a$. Finally, the Alamouti transform of a $K \times 2$ matrix $\boldsymbol{X}=\left[\begin{array}{ll}\boldsymbol{x}_{1} & \boldsymbol{x}_{2}\end{array}\right]$ is defined as $\mathcal{A}(\boldsymbol{X}) \triangleq\left[-\boldsymbol{x}_{2}^{*} \boldsymbol{x}_{1}^{*}\right]$.

\section{MIMO GO-CDM system model}

We consider a MIMO multicarrier system with $N_{c}$ data subcarriers, equipped with $N_{T}$ and $N_{R}$ transmit and receive antennas, respectively, and configured to transmit $N_{s}\left(\leq N_{T}\right)$ spatial data streams. Following the grouporthogonal design principles, the available subcarriers are split into $N_{g}=N_{c} / Q$ groups of $Q$ subcarriers each. In the following subsections the transmitter, channel model and reception equation are described in detail.

\subsection{Transmitter}

As depicted in Figure 1, incoming bits are split into $N_{s}$ spatial streams, which are then processed separately. Bits on the $z$ th stream are mapped onto a sequence $s^{z}$ of symbols drawn from an $M$-ary complex constellation (e.g., BPSK, M-QAM) with average normalized unit energy. The resulting $N_{s}$ streams of modulated symbols $\left\{\boldsymbol{s}^{z}\right\}_{z=1}^{N_{s}}$ are then fed to the GO-CDM stage, which comprises three steps:

1. Segmentation of the incoming symbol stream in blocks of length $N_{c}$ (i.e., eventual OFDM symbols), and serial to parallel conversion (S/P) resulting, over the $k$ th OFDM symbol period, in $\boldsymbol{s}^{z}(k)$.
2. Arrangement of the symbols in the block into groups $\left\{s_{g}^{z}(k)\right\}_{g=1}^{N_{g}}$, where $\boldsymbol{s}_{g}^{z}(k)=\left[s_{g, 1}^{z}(k) \ldots s_{g, Q}^{z}(k)\right]^{T}$ represents an individual group.

3. Group spreading through a linear combination

$$
\tilde{\boldsymbol{s}}_{g}^{z}(k)=\frac{1}{\sqrt{N_{T}}} \boldsymbol{C} \boldsymbol{s}_{g}^{z}(k),
$$

where $C$ is a $Q \times Q$ orthonormal matrix, typically chosen to be a rotated Walsh-Hadamard matrix [4].

Before the usual OFDM modulation steps on each antenna (IFFT, guard interval appending and upconversion), the grouped and spread symbols are processed in accordance with the MIMO transmission scheme in use as follows:

\subsection{1 $\operatorname{SDM}\left(N_{s}=N_{T}\right)$}

In this case the blocks labeled in Figure 1 as STBC and CDD are not used, and the spread symbols are directly supplied to the antenna mapping stage, which simply connects the incoming $z$ th data stream to the $i$ th transmit branch $\left(1 \leq i \leq N_{T}\right)$, that is,

$$
\breve{s}_{g}^{i}(k)=\hat{\boldsymbol{s}}_{g}^{i}(k)=\tilde{\boldsymbol{s}}_{g}^{z}(k) .
$$

2.1.2 $\operatorname{STBC}\left(N_{s}=1, N_{T}=2\right)$

Two consecutive blocks of spread symbols, $\tilde{\boldsymbol{s}}_{g}^{1}(k)$ and $\tilde{\boldsymbol{s}}_{g}^{1}(k+1)$, are Alamouti-encoded on a per-subcarrier basis over two OFDM symbol periods,

$$
\begin{aligned}
& \hat{\boldsymbol{s}}_{g}^{1}(k)=\tilde{\boldsymbol{s}}_{g}^{1}(k), \quad \hat{\boldsymbol{s}}_{g}^{1}(k+1)=-\left(\tilde{\boldsymbol{s}}_{g}^{1}(k+1)\right)^{*}, \\
& \hat{\boldsymbol{s}}_{g}^{2}(k)=\tilde{\boldsymbol{s}}_{g}^{1}(k+1), \hat{\boldsymbol{s}}_{g}^{2}(k+1)=\left(\tilde{\boldsymbol{s}}_{g}^{1}(k)\right)^{*} .
\end{aligned}
$$

In the antenna mapping stage, STBC-encoded streams are connected to two transmit branches, one for each symbol of the STBC code, that is,

$$
\breve{s}_{g}^{i}(k)=\hat{\boldsymbol{s}}_{g}^{i}(k)
$$

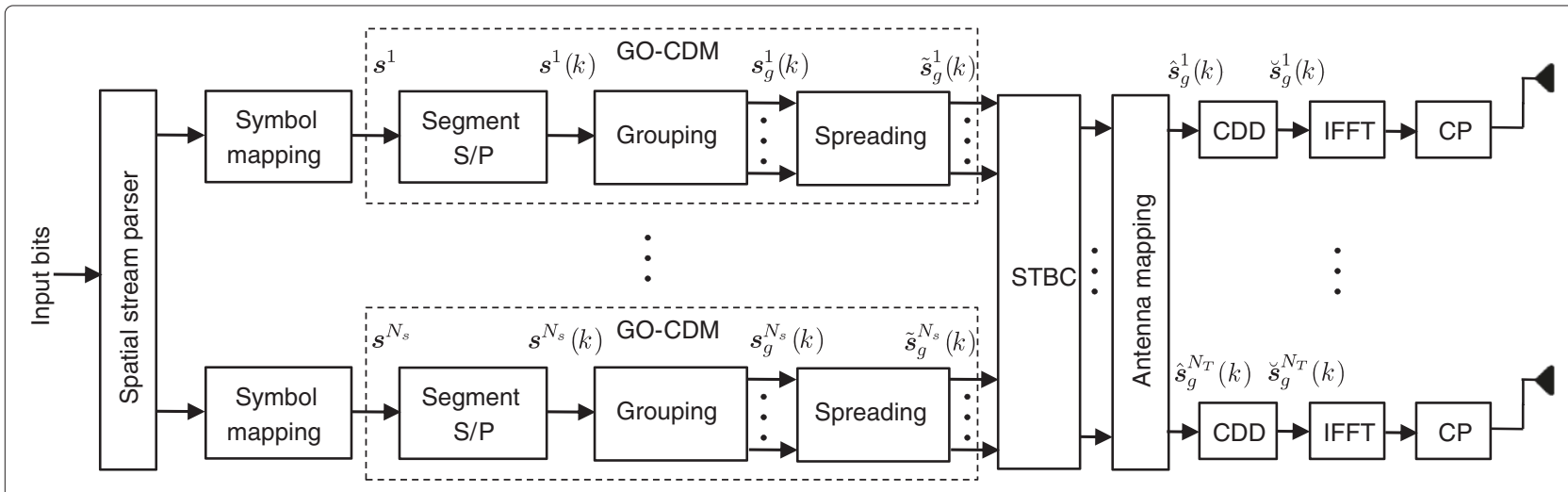

Figure 1 Transmitter architecture for MIMO GO-CDM. 


\subsection{3 $C D D\left(N_{s}=1\right)$}

In a pure CDD scheme, the same data stream is sent through $N_{T}$ antennas with each replica being subject to a different cyclic delay $\Delta_{i}$, typically chosen as $\Delta_{i}=\Delta_{i-1}+$ $N_{c} / N_{T}$ with $\Delta_{1}=0$ [15], resulting in transmitted symbols

$$
\breve{s}_{g, q}^{i}(k)=\tilde{s}_{g, q}^{1}(k) \exp \left(-j 2 \pi d_{q} \Delta_{i} / N_{c}\right),
$$

where $d_{q}$ denotes the subcarrier index.

\subsubsection{Hybrid schemes}

The analytical framework developed in this paper can also be applied to hybrid systems combining SDM, STBC and/or CDD. Nevertheless, for brevity of presentation, the analysis to be developed next focuses on scenarios where only one of the mechanisms is used.

\subsection{Channel model}

The channel linking an arbitrary pair of Tx and Rx antennas is assumed to be time-varying and frequency-selective with an scenario-dependent power delay profile

$$
S(\tau)=\sum_{l=0}^{P-1} \phi_{l} \delta\left(\tau-\tau_{l}\right)
$$

where $P$ denotes the number of independent paths of the channel and $\phi_{l}$ and $\tau_{l}$ denote the power and delay of the $l$-th path. It is assumed that the power delay profile is the same for all pairs of $\mathrm{Tx}$ and $\mathrm{Rx}$ antennas and that it has been normalized to unity (i.e., $\sum_{l=0}^{P-1} \phi_{l}=1$ ). A single realization of the channel impulse response between $\mathrm{Tx}$ antenna $i$ and receive antenna $j$ at time instant $t$ will then have the form

$$
h^{i j}(t ; \tau)=\sum_{l=0}^{P-1} h_{l}^{i j}(t) \delta\left(\tau-\tau_{l}\right)
$$

where it will hold that $E\left\{\left|h_{l}^{i j}(t)\right|^{2}\right\}=\phi_{l}$. The corresponding frequency response can be expressed as

$$
\bar{h}^{i j}(t ; f)=\sum_{l=0}^{P-1} h_{l}^{i j}(t) \exp \left(-j 2 \pi f \tau_{l}\right),
$$

which when evaluated at the $N_{c}$ OFDM subcarriers yields

$$
\overline{\boldsymbol{h}}^{i j}(t)=\left[\bar{h}^{i j}\left(t ; f_{0}\right) \ldots \bar{h}^{i j}\left(t ; f_{N_{c}-1}\right)\right]^{T} .
$$

In order to simplify the notation, assuming that the channel is static over the duration of a block (i.e., an OFDM symbol), the frequency response between Tx-antenna $i$ and Rx-antenna $j$ over the $N_{c}$ subcarriers during the kth OFDM symbol can be expressed as

$$
\overline{\boldsymbol{h}}^{i j}(k)=\left[\bar{h}_{0}^{i j}(k) \ldots \bar{h}_{N_{c}-1}^{i j}(k)\right]^{T} .
$$

Since the subsequent analysis is mostly conducted on a per-group basis, the channel frequency response for the $g$ th group is denoted by

$$
\overline{\boldsymbol{h}}_{g}^{i j}(k)=\left[\bar{h}_{g, 1}^{i j}(k) \ldots \bar{h}_{g, Q}^{i j}(k)\right]^{T},
$$

with correlation matrix given by

$$
\boldsymbol{\mathcal { R }}_{\boldsymbol{h}_{g}}=E\left\{\left\|\overline{\boldsymbol{h}}_{g}^{i j}(k)\right\|^{2}\right\}=E\left\{\overline{\boldsymbol{h}}_{g}^{i j}(k)\left(\overline{\boldsymbol{h}}_{g}^{i j}(k)\right)^{H}\right\},
$$

which is assumed to be constant over time, common for all pairs of $\mathrm{Tx}$ and $\mathrm{Rx}$ antennas and, provided that group subcarriers are chosen evenly within the available bandwidth, common to all groups. In order to maximise the frequency diversity gain, subcarriers forming a group should be chosen equispaced across the available bandwidth when employing SDM or STBC. In contrast, when using CDD, subcarriers should be grouped taking into account that, due to the CDD action, an arbitrary subcarrier is totally uncorrelated with the following $N_{T}-1$ subcarriers [15].

Now, considering the spatial correlation introduced by the transmit and receive antenna arrays, the spatially correlated channel frequency response for an arbitrary subcarrier $q$ in group $g$ can be expressed as [16]

$$
\mathcal{H}_{g, q}(k)=\boldsymbol{\mathcal { R }}_{R X}^{1 / 2} \boldsymbol{H}_{g, q}(k)\left(\boldsymbol{\mathcal { R }}_{T X}^{1 / 2}\right)^{T},
$$

where $\boldsymbol{\mathcal { R }}_{R X}$ and $\boldsymbol{\mathcal { R }}_{T X}$ are, respectively, $N_{R} \times N_{R}$ and $N_{T} \times N_{T}$ matrices denoting the receive and transmit correlation, and

$$
\boldsymbol{H}_{g, q}(k)=\left(\begin{array}{ccc}
\bar{h}_{g, q}^{11}(k) & \ldots & \bar{h}_{g, q}^{1 N_{T}}(k) \\
\vdots & & \vdots \\
\bar{h}_{g, q}^{N_{R} 1}(k) & \ldots & \bar{h}_{g, q}^{N_{R} N_{T}}(k)
\end{array}\right) .
$$

\subsubsection{Receiver}

As shown in Figure 2, the reception process begins by removing the cyclic prefix and performing an FFT to recover the symbols in the frequency domain. After $\mathrm{S} / \mathrm{P}$ conversion, and assuming ideal synchronization at the receiver side, the received samples for group $g$ at the output of the FFT processing stage can be expressed in accordance with the MIMO transmission scheme in use as follows:

1) SDM and CDD: In these cases,

$$
\boldsymbol{r}_{g}(k)=\operatorname{vec}\left(\left[\boldsymbol{r}_{g, 1}(k) \ldots \boldsymbol{r}_{g, Q}(k)\right]\right)=\mathcal{H}_{g}(k) \breve{s}_{g}(k)+\boldsymbol{v}_{g}(k),
$$

where the $N_{R} Q \times N_{T} Q$ matrix

$$
\mathcal{H}_{g}(k)=\mathcal{D}\left(\left[\mathcal{H}_{g, 1}(k) \ldots \mathcal{H}_{g, Q}(k)\right]\right),
$$

represents the spatially and frequency correlated channel matrix affecting all symbols transmitted in 


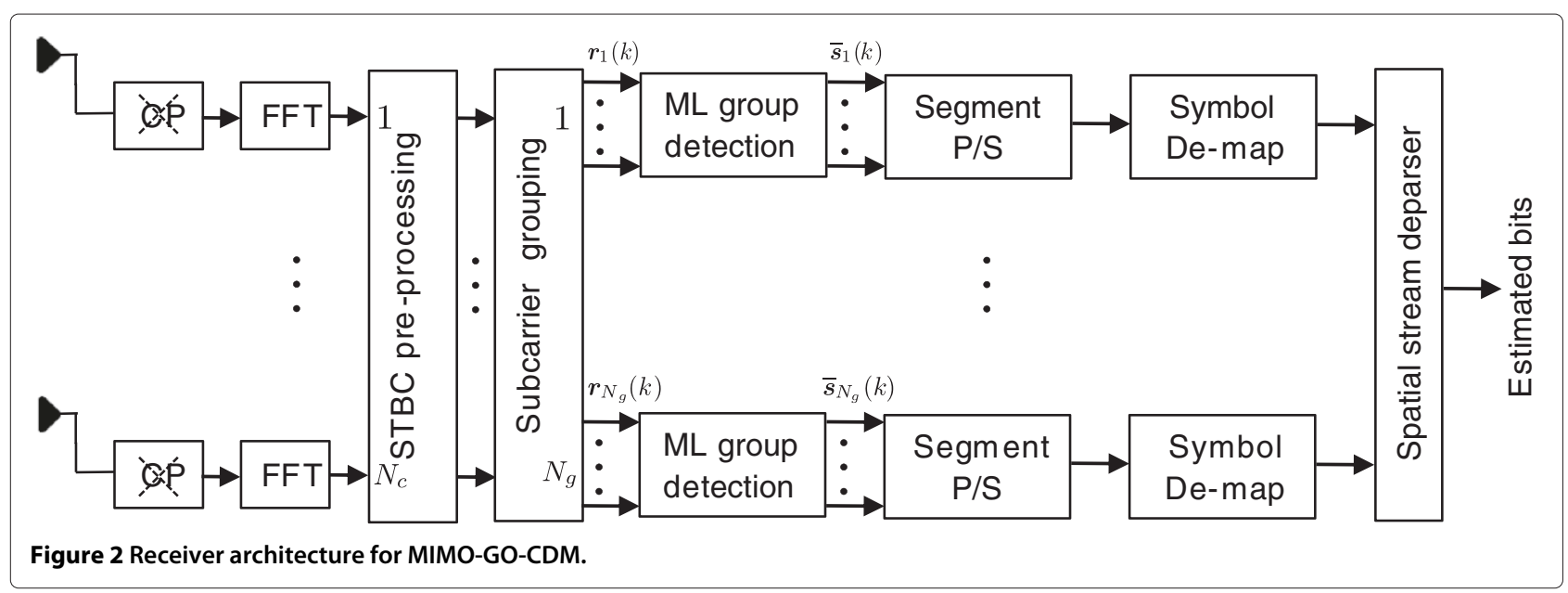

group g, the $N_{s} Q$-long vector of transmitted (spread) symbols is formed as

$$
\breve{s}_{g}(k)=\operatorname{vec}\left(\left[\breve{s}_{g}^{1}(k) \ldots \breve{s}_{g}^{N_{T}}(k)\right]^{T}\right),
$$

and finally, $\boldsymbol{v}_{g}(k)$ is an $N_{R} Q \times 1$ vector representing the receiver noise, with each component being drawn from a circularly symmetric zero-mean white Gaussian distribution with variance $\sigma_{v}^{2}$.

2) STBC: As stated in (3), STBC encoding period $\eta=k / 2$, with $k=0,2,4, \ldots$, spawns two consecutive OFDM symbol periods, namely, the $k$ th and $(k+1)$ th symbol periods. Assuming that the channel coherence time is large enough to safely consider that $\mathcal{H}_{g}(k+1)=\mathcal{H}_{g}(k)$, then,

$$
\begin{aligned}
& \tilde{\boldsymbol{r}}_{g}(k)=\boldsymbol{\mathcal { H }}_{g}(k) \breve{\boldsymbol{s}}_{g}(k)+\boldsymbol{v}_{g}(k), \\
& \tilde{\boldsymbol{r}}_{g}(k+1)=\mathcal{H}_{g}(k) \breve{\boldsymbol{s}}_{g}(k+1)+\boldsymbol{v}_{g}(k+1),
\end{aligned}
$$

and, therefore, we can define an equivalent received vector in STBC encoding period $\eta$ as

$$
\begin{aligned}
\boldsymbol{r}_{g}(\eta) \triangleq\left[\begin{array}{c}
\tilde{\boldsymbol{r}}_{g}(k) \\
\tilde{\boldsymbol{r}}_{g}^{*}(k+1)
\end{array}\right] & =\left[\begin{array}{c}
\mathcal{H}_{g}(k) \\
\mathcal{H}_{g}^{\mathcal{A}}(k)
\end{array}\right] \tilde{\boldsymbol{s}}_{g}(\eta)+\left[\begin{array}{c}
\boldsymbol{v}_{g}(k) \\
\boldsymbol{v}_{g}^{*}(k+1)
\end{array}\right] \\
& \triangleq \tilde{\mathcal{H}}_{g}(\eta) \tilde{\boldsymbol{s}}_{g}(\eta)+\tilde{\boldsymbol{v}}_{g}(\eta),
\end{aligned}
$$

where

$$
\mathcal{H}_{g}^{\mathcal{A}}(k) \triangleq \mathcal{D}\left(\left[\mathcal{A}\left(\mathcal{H}_{g, 1}(k)\right) \ldots \mathcal{A}\left(\mathcal{H}_{g, Q}(k)\right)\right]\right)
$$

and

$$
\tilde{\boldsymbol{s}}_{g}(\eta) \triangleq \operatorname{vec}\left(\left[\tilde{\boldsymbol{s}}_{g}^{1}(k) \tilde{\boldsymbol{s}}_{g}^{1}(k+1)\right]^{T}\right) .
$$

In order to facilitate the unified performance analysis of the different MIMO strategies, it is more convenient to express the reception equation in terms of the original symbols rather than the spread ones. Thus, defining

$$
\begin{array}{ll}
\boldsymbol{s}_{g}(k)=\frac{1}{\sqrt{N_{T}}} \operatorname{vec}\left(\left[\boldsymbol{s}_{g}^{1}(k) \ldots \boldsymbol{s}_{g}^{N_{s}}(k)\right]^{T}\right) & \mathrm{SDM} \\
\boldsymbol{s}_{g}(\eta)=\frac{1}{\sqrt{2}} \operatorname{vec}\left(\left[\boldsymbol{s}_{g}^{1}(k) \boldsymbol{s}_{g}^{1}(k+1)\right]^{T}\right) & \mathrm{STBC} \\
\boldsymbol{s}_{g}(k)=\frac{1}{\sqrt{N_{T}}} \boldsymbol{s}_{g}^{1}(k) & \mathrm{CDD}
\end{array}
$$

it is straightforward to check that the symbols to be supplied to the IFFT processing step are given by,

$$
\begin{array}{ll}
\breve{\boldsymbol{s}}_{g}(k)=\left(\boldsymbol{C} \otimes \boldsymbol{I}_{N_{s}}\right) \boldsymbol{s}_{g}(k) & \mathrm{SDM} \\
\breve{\boldsymbol{s}}_{g}(k)=\tilde{\boldsymbol{s}}_{g}(\eta)=\left(\boldsymbol{C} \otimes \boldsymbol{I}_{2}\right) \boldsymbol{s}_{g}(\eta) & \mathrm{STBC} \\
\breve{\boldsymbol{s}}_{g}(k)=\boldsymbol{E}_{g}^{\Delta}\left(\boldsymbol{C} \otimes \mathbf{1}_{N_{T} \times 1}\right) \boldsymbol{s}_{g}(k) & \mathrm{CDD}
\end{array}
$$

where $\boldsymbol{E}_{g}^{\Delta} \triangleq \mathcal{D}\left(\left[\boldsymbol{E}_{g}^{\Delta 1} \ldots \boldsymbol{E}_{g}^{\Delta Q}\right]\right)$ is a block diagonal matrix with elements $\boldsymbol{E}_{g}^{\Delta q}=\mathcal{D}\left(\left[e^{-j 2 \pi d_{q} \Delta_{1} / N_{c}} \ldots\right.\right.$ $\left.\left.e^{-j 2 \pi d_{q} \Delta_{N_{T}} / N_{c}}\right]\right)$ [15]. Furthermore, since processing takes place either on an OFDM symbol basis for SDM and CDD systems or on an STBC encoding period basis for STBC schemes, the indexes $k$ and/or $\eta$ can be dropped from this point onwards, allowing the reception equation to be expressed in general form as

$$
\boldsymbol{r}_{g}=\boldsymbol{A}_{g} \boldsymbol{s}_{g}+\boldsymbol{v}_{g}
$$

where

$$
\boldsymbol{A}_{g}= \begin{cases}\mathcal{H}_{g}\left(\boldsymbol{C} \otimes \boldsymbol{I}_{N_{s}}\right) & \text { SDM } \\ \tilde{\mathcal{H}}_{g}\left(\boldsymbol{C} \otimes \boldsymbol{I}_{2}\right) & \text { STBC } \\ \mathcal{H}_{g} \boldsymbol{E}_{g}^{\Delta}\left(\boldsymbol{C} \otimes \mathbf{1}_{N_{T} \times 1}\right) & \text { CDD }\end{cases}
$$

and

$$
\boldsymbol{v}_{g}=\left\{\begin{array}{ll}
\boldsymbol{v}_{g} & \mathrm{SDM} / \mathrm{CDD} \\
\tilde{\boldsymbol{v}}_{g} & \mathrm{STBC}
\end{array} .\right.
$$

It should be noted that, regardless of the MIMO scheme and group dimension in use, the system matrix $\boldsymbol{A}_{g}$ has 
been normalised such that the SNR can be defined as $E_{s} / N_{0}=1 /\left(2 \sigma_{v}^{2}\right)$.

Upon reception, all symbols in a group (for all streams in SDM and for both encoded OFDM symbols in STBC) are jointly estimated using an ML detection process. That is, the vector of estimated symbols in a group can be expressed as

$$
\overline{\boldsymbol{s}}_{g}=\arg \min _{\boldsymbol{s}_{g}}\left\|\boldsymbol{A}_{g} \boldsymbol{s}_{g}-\boldsymbol{r}_{g}\right\|^{2} .
$$

This procedure amounts to evaluate all the possible transmitted vectors and choosing the closest one (in a leastsquares sense) to the received vector. Nevertheless, sphere detection [17] can be used for efficiently performing the exhaustive search required to implement the ML estimation.

\section{Unified bit error rate analysis}

\subsection{BER analysis based on pairwise error probability}

Using the well-known union bound [18], which is very tight for high signal-to-noise ratios, the bit error probability can be upper bounded as

$$
\begin{aligned}
& P_{b} \leq \frac{1}{N_{g} N_{Q} M^{N_{Q}} \log _{2} M} \sum_{g=1}^{N_{g}} \sum_{u=1}^{M^{N_{Q}}} \sum_{\substack{w=1 \\
w \neq u}}^{M^{N_{Q}}} P \\
& \times\left(\boldsymbol{s}_{g, u} \rightarrow \boldsymbol{s}_{g, w}\right) \mathcal{N}_{b}\left(\boldsymbol{s}_{g, u}, \boldsymbol{s}_{g, w}\right),
\end{aligned}
$$

where the expression $P\left(\boldsymbol{s}_{g, u} \rightarrow \boldsymbol{s}_{g, w}\right)$, usually called the pairwise error probability (PEP), represents the probability of erroneously detecting the vector $\boldsymbol{s}_{g, w}$ when $\boldsymbol{s}_{g, u}$ was transmitted and $\mathcal{N}_{b}\left(\boldsymbol{s}_{g, u}, \boldsymbol{s}_{g, w}\right)$ is equal to the number of differing bits between vectors $\boldsymbol{s}_{g, u}$ and $\boldsymbol{s}_{g, w}$ and $N_{Q}$ is defined as,

$$
N_{Q}=\left\{\begin{array}{cc}
N_{s} & \text { SDM } \\
2 Q & \text { STBC } \\
Q & \text { CDD }
\end{array}\right.
$$

To proceed further, the PEP conditioned on $\boldsymbol{A}_{g}$ can be shown to be [19]

$$
\begin{aligned}
P\left(\boldsymbol{s}_{g, u} \rightarrow \boldsymbol{s}_{g, w} \mid \boldsymbol{A}_{g}\right)= & \frac{1}{2} \operatorname{erfc}\left(\sqrt{\frac{\left\|\boldsymbol{A}_{g}\left(\boldsymbol{s}_{g, u}-\boldsymbol{s}_{g, w}\right)\right\|^{2}}{4 \sigma_{v}^{2}}}\right) \\
= & \frac{1}{\pi} \int_{0}^{\pi / 2} \exp \left(-\frac{\left\|\boldsymbol{A}_{g}\left(\boldsymbol{s}_{g, u}-\boldsymbol{s}_{g, w}\right)\right\|^{2}}{4 \sigma_{v}^{2} \sin ^{2} \phi}\right) \\
& \times d \phi .
\end{aligned}
$$

Now, defining the random variable $d_{g, u w}^{2} \triangleq \| \boldsymbol{A}_{g}\left(\boldsymbol{s}_{g, u}-\right.$ $\left.\boldsymbol{s}_{g, w}\right) \|^{2}$, the average PEP can be obtained as

$$
\begin{aligned}
P\left(s_{g, u} \rightarrow \boldsymbol{s}_{g, w}\right) & =\frac{1}{\pi} \int_{0}^{\pi / 2} \int_{-\infty}^{+\infty} e^{-x / 4 \sigma_{v}^{2} \sin ^{2} \phi} p_{d_{g, u w}^{2}}(x) d x d \phi \\
& =\frac{1}{\pi} \int_{0}^{\pi / 2} \mathcal{M}_{d_{g, u w}^{2}}\left(-\frac{1}{4 \sigma_{v}^{2} \sin ^{2} \phi}\right) d \phi,
\end{aligned}
$$

where $p_{x}(\cdot)$ and $\mathcal{M}_{x}(\cdot)$ denote the probability density function (pdf) and moment generating function (MGF) of a random variable $x$, respectively.

Let us now define the error vector $\boldsymbol{e}_{g, u w}=\boldsymbol{s}_{g, u}-\boldsymbol{s}_{g, w}$. Using this definition, it can be shown that

$$
d_{g, u w}^{2} \triangleq\left\|\boldsymbol{A}_{g} \boldsymbol{e}_{g, u w}\right\|^{2}=\mathcal{H}_{g}^{H} \boldsymbol{T}_{g, u w}^{H} \boldsymbol{T}_{g, u w} \mathcal{H}_{g},
$$

where

$$
\mathcal{H}_{g} \triangleq \operatorname{vec}\left[\operatorname{vec}\left(\mathcal{H}_{g, 1}\right) \ldots \operatorname{vec}\left(\mathcal{H}_{g, Q}\right)\right],
$$

and $\boldsymbol{T}_{g, u w}$ can be expressed as

$$
\boldsymbol{T}_{g, u w}= \begin{cases}{\left[\left(\mathbf{1}_{Q \times 1} \otimes \boldsymbol{S}_{g, u w}\right) \odot \mathfrak{I}_{Q, N_{T}}\right] \otimes \boldsymbol{I}_{N_{R}}} & \mathrm{SDM} / \mathrm{CDD} \\ {\left[\left(\mathbf{1}_{1 \times Q} \otimes \boldsymbol{S}_{g, u w}^{T}\right) \odot \mathfrak{I}_{Q, 2}^{T}\right] \otimes \boldsymbol{I}_{2 N_{R}}} & \mathrm{STBC}\end{cases}
$$

with

$$
\boldsymbol{S}_{g, u w}= \begin{cases}\boldsymbol{e}_{g, u w}^{T}\left(\boldsymbol{C}^{T} \otimes \boldsymbol{I}_{N_{T}}\right) & \mathrm{SDM} / \mathrm{STBC} \\ \boldsymbol{e}_{g, u w}^{T}\left(\boldsymbol{C}^{T} \otimes \mathbf{1}_{1 \times N_{T}}\right) \boldsymbol{E}_{\Delta}^{T} & \mathrm{CDD}\end{cases}
$$

and $\mathfrak{I}_{n, m} \triangleq \boldsymbol{I}_{n} \otimes \mathbf{1}_{1 \times m}$. The expression of $d_{g, u w}^{2}$ reveals that it is a quadratic form in complex variables $\mathcal{H}_{g}$, with MGF given by

$$
\mathcal{M}_{d_{g, u w}^{2}}(s)=\left|\boldsymbol{I}_{N}-s \boldsymbol{G}_{g, u w}\right|^{-1},
$$

where $N$ is equal to $Q N_{R}$ for the SDM and CDD schemes, and equal to $4 Q N_{R}$ for the STBC strategy. Furthermore,

$$
\boldsymbol{G}_{g, u w}=\boldsymbol{T}_{g, u w} \boldsymbol{R}_{g} \boldsymbol{T}_{g, u w}^{H},
$$

with

$$
\boldsymbol{R}_{g}=\boldsymbol{\mathcal { R }}_{\boldsymbol{h}_{g}} \otimes \boldsymbol{\mathcal { R }}_{T X} \otimes \mathcal{R}_{R X} .
$$

Now, let $\lambda_{g, u w}=\left\{\lambda_{g, u w, 1}, \ldots, \lambda_{g, u w, D_{g, u w}}\right\}$ denote the set of $D_{g, u w}$ distinct positive eigenvalues of $\boldsymbol{G}_{g, u w}$ with corresponding multiplicities $\boldsymbol{\alpha}_{g, u w}=\left\{\alpha_{g, u w, 1}, \ldots, \alpha_{g, u w, D_{g, u w}}\right\}$. Using the results in [20], the MGF of $d_{g, u w}^{2}$ can also be expressed as

$$
\begin{aligned}
\mathcal{M}_{d_{g, u w}^{2}}(s) & =\prod_{d=1}^{D_{g, u w}} \frac{1}{\left(1-s \lambda_{g, u w, d}\right)^{\alpha_{g, u w, d}}} \\
& =\sum_{d=1}^{D_{g, u w}} \sum_{p=1}^{\alpha_{g, u w, d}} \frac{\kappa_{g, u w, d, p}}{\left(1-s \lambda_{g, u w, d}\right)^{p}}
\end{aligned}
$$


where, using ([21], Theorem 1), it can be shown that

$$
\kappa_{g, u w, d, p}=\lambda_{g, u w, d}^{p-\alpha_{g, u w, d}} \sum_{\Phi} \prod_{\substack{d^{\prime}=1 \\
d^{\prime} \neq d}}^{D_{g, u w}} \frac{\lambda_{g, u w, d^{\prime}}^{n_{d^{\prime}}}\left(\begin{array}{c}
\alpha_{g, u w, d^{\prime}}+n_{d^{\prime}}-1 \\
n_{d^{\prime}}
\end{array}\right)}{\left(1-\frac{\lambda_{g, u w, d^{\prime}}}{\lambda_{g, u w, d}}\right)^{\alpha_{g, u w, d^{\prime}}+n_{d^{\prime}}}}
$$

with $\Phi$ being the set of nonnegative integers $\left\{n_{1}, \ldots, n_{d-1}, n_{d+1}, \ldots, n_{D_{g, u w}}\right\}$ such that $\sum_{d^{\prime} \neq d} n_{d^{\prime}}=$ $\alpha_{g, u w, d}-p$, which allows (28) to be written as

$$
\begin{aligned}
P\left(\boldsymbol{s}_{g, u} \rightarrow \boldsymbol{s}_{g, w}\right)= & \frac{1}{\pi} \sum_{d=1}^{D_{g, u w}} \sum_{p=1}^{\alpha_{g, u w, d}} \kappa_{g, u w, d, p} \int_{0}^{\pi / 2} \\
& \times\left(\frac{\sin ^{2} \phi}{\sin ^{2} \phi+\frac{\lambda_{g, u w, d}}{4 \sigma_{v}^{2}}}\right)^{p} d \phi \\
= & \sum_{d=1}^{D_{g, u w}} \sum_{p=1}^{\alpha_{g, u w, d}} \kappa_{g, u w, d, p}\left(\frac{1-\Omega\left(\frac{\lambda_{g, u w, d}}{4 \sigma_{v}^{2}}\right)}{2}\right) p \\
& \times \sum_{g=0}^{p-1}\left(\begin{array}{c}
p-1+g \\
g
\end{array}\right)\left(\frac{1+\Omega\left(\frac{\lambda_{g, u w, d}}{4 \sigma_{v}^{2}}\right)}{2}\right) g,
\end{aligned}
$$

with $\Omega(c)=\sqrt{c /(1+c)}$. By substituting (38) into (25), a closed-form BER upper bound for an arbitrary power delay profile is obtained. It is later shown that this bound is tight, accurately matching the simulation results.

\subsection{BER analysis based on PEP classes}

Note that many pairs $\left(\boldsymbol{s}_{g, u}, \boldsymbol{s}_{g, w}\right)$ result in exactly the same PEP, allowing the definition of a pairwise error class $\mathcal{C}_{g, c} \triangleq$ $\mathcal{C}\left(D_{g, c}, \lambda_{g, c}, \boldsymbol{\alpha}_{g, c}\right)$ as the set of all pairs $\left(\boldsymbol{s}_{g, u}, \boldsymbol{s}_{g, w}\right)$ characterized by a common matrix $\boldsymbol{G}_{g, u w}=\boldsymbol{G}_{g, c}$ with $D_{g, c}$ distinct eigenvalues $\lambda_{g, c}=\left\{\lambda_{g, c, 1}, \ldots, \lambda_{g, c, D_{g, c}}\right\}$ with corresponding multiplicities $\boldsymbol{\alpha}_{g, c}=\left\{\alpha_{g, c, 1}, \ldots, \alpha_{g, c, D_{g, c}}\right\}$ and therefore, a common PEP denoted by $\mathcal{P}\left(D_{g, c}, \boldsymbol{\lambda}_{g, c}, \boldsymbol{\alpha}_{g, c}\right)$. For each class, the scalar $W\left(D_{g, c}, \lambda_{g, c}, \boldsymbol{\alpha}_{g, c}, \mathcal{N}\right)$ represents the number of elements in the class $\mathcal{C}_{g, c}$ inducing $\mathcal{N}$ erroneous bits. A more insightful BER expression can then be obtained by using the PEP class notation, avoiding in this way the exhaustive computation of all the PEPs. Instead, the BER upper-bound can be found by computing the PEP for each class and weighing it using the number of elements in the class and the number of erroneous bits this class may induce. The BER upper bound can then be rewritten as

$$
\begin{aligned}
P_{b} \leq & \frac{1}{N_{g} N_{Q} M^{N_{Q}} \log _{2} M} \\
& \times \sum_{g=1}^{N_{g}} \sum_{\forall \mathcal{C}_{g, c}} \sum_{\mathcal{N}=1}^{N_{Q} \log _{2} M} \mathcal{N} W\left(D_{g, c}, \lambda_{g, c}, \boldsymbol{\alpha}_{g, c}, \mathcal{N}\right) \\
& \times \mathcal{P}\left(D_{g, c}, \lambda_{g, c}, \boldsymbol{\alpha}_{g, c}\right)
\end{aligned}
$$

where $W\left(D_{g, c}, \lambda_{g, c}, \boldsymbol{\alpha}_{g, c}, \mathcal{N}\right)$ corresponds to the number of elements in the class $\mathcal{C}_{g, c}$ inducing $\mathcal{N}$ erroneous bits.

\subsection{Asymptotic performance}

Further insight on the parameters affecting the BER performance can be obtained by focusing on the asymptotic case of large SNR. When $E_{s} / N_{0} \rightarrow \infty$, the argument of the MGF in (28) also tends to infinity, thus allowing the asymptotic PEP of the different classes to be expressed as [4],

$$
\begin{aligned}
\mathcal{P}_{\text {asym }}\left(D_{g, c}, \lambda_{g, c}, \boldsymbol{\alpha}_{g, c}\right) & =\frac{1}{\pi} \int_{0}^{\pi / 2} \frac{\left(4 \sigma_{v}^{2} \sin ^{2} \phi\right)^{\tilde{D}_{g, c}}}{\prod_{d=1}^{D_{g, c}} \lambda_{g, c, c, d}^{\alpha_{g}}} d \phi \\
& =\frac{\left(2 \tilde{D}_{g, c}\right) !}{2 \tilde{D}_{g, c} !^{2}} \frac{\left(E_{s} / N_{0}\right)^{-\tilde{D}_{g, c}}}{\prod_{d=1}^{\tilde{D}_{\min }} \lambda_{g, c, d}^{\alpha_{g, c, d}}},
\end{aligned}
$$

where $\tilde{D}_{g, c}=\sum_{d=1}^{D_{g, c}} \alpha_{g, c, d}$ is the rank of the matrixdefining class $\boldsymbol{G}_{g, c}$. From (40) it is clear that the probability of error will be mainly determined by the groups and classes whose matrices

$$
\boldsymbol{G}_{g, c}=\boldsymbol{G}_{g, c}^{\min } \triangleq \boldsymbol{T}_{g, c}^{\min } \boldsymbol{R}_{g}^{\min }\left(\boldsymbol{T}_{g, c}^{\min }\right)^{H}
$$

have the smallest common rank, denoted by

$$
\tilde{D}_{\min }=\operatorname{rank}\left(\boldsymbol{G}_{g, c}^{\min }\right)=\operatorname{rank}\left(\boldsymbol{T}_{g, c}^{\min } \boldsymbol{R}_{g}^{\min }\left(\boldsymbol{T}_{g, c}^{\min }\right)^{H}\right) .
$$

From (40), it can be deducted that the asymptotic BER minimisation is achieved by maximising the minimum group/class rank $\tilde{D}_{\min }$ and the eigenvalue product of all the groups/classes with rank $\tilde{D}_{\text {min }}$. In the following, only the maximization of $\tilde{D}_{\min }$ (i.e., maximisation of the diversity order) is pursued since the maximization of the product of eigenvalues is far more difficult as it involves the simultaneous optimization of all the eigenvalue products in the groups/classes with $\operatorname{rank} \tilde{D}_{\text {min }}$.

\subsubsection{On the rank of $T_{g, c}^{\min }$}

As mentioned in Section 2.2, choosing the subcarriers forming a group in an appropriate manner (and in 
accordance with the selected MIMO mode) minimizes subcarrier correlation allowing the optimization of the system performance if an adequate family of spreading codes is properly selected. To this end, rotated spreading transforms have been proposed for multicarrier systems in [22] where it is shown that the often used Walsh-Hadamard codes lead to poor diversity gains when employed to perform the frequency spreading. This can be explained by the fact that for certain symbol blocks the energy is concentrated on one single subcarrier and, thus,

$$
\operatorname{rank}\left(\boldsymbol{T}_{g, c}^{\min }\right)= \begin{cases}N_{R} & \mathrm{SDM} \\ N_{T} N_{R} & \mathrm{STBC} / \mathrm{CDD}\end{cases}
$$

A deep fade on this subcarrier dramatically raises the probability of error in the detection process, regardless of the state of all other subcarriers, limiting in this way the achievable diversity order (asymptotic BER slope). A similar effect can be observed when using other spreading sequences such as those based on the discrete Fourier transform (DFT). As pointed out in [22], a spreading that has the potential to maximize the diversity order can be found by applying a rotation to the columns of the conventional spreading matrix $C_{\text {conv }}$ as $\boldsymbol{C}=\boldsymbol{C}_{\text {conv }} \mathcal{D}(\boldsymbol{\theta})$, where $\boldsymbol{\theta}=\left[\theta_{1} \ldots \theta_{Q}\right]$ with each $\theta_{q}$ denoting the chip-specific rotation, which in the proposed scheme is given by

$$
\theta_{q}=\exp \left(\frac{j 2 \pi(q-1)}{Q \Theta}\right)
$$

with $\Theta$ being constellation dependent and selected so as to make $2 \pi / \Theta$ the minimum angle producing a rotation of the transmit symbol alphabet onto itself (e.g., $\Theta=2$ for BPSK, $\Theta=4$ for MQAM). This indicates that while using conventional Walsh-Hadamard spreading no frequency diversity gain will be achieved, the a rotation of the spreading matrix such as the ones defined by (3.3.1) has the potential (depending on the channel correlation matrix $\boldsymbol{R}_{g}$ ) to attain a frequency diversity gain proportional to the number of subcarriers per group, common to all groups and classes. That is, when using optimally rotated spreading codes,

$$
\operatorname{rank}\left(T_{g, c}^{\min }\right)= \begin{cases}Q N_{R} & \mathrm{SDM} \\ Q N_{T} N_{R} & \mathrm{STBC} / \mathrm{CDD}\end{cases}
$$

\subsubsection{On the rank of $R_{g}^{\min }$}

The correlation matrix $\boldsymbol{R}_{g}^{\min }$ can be expressed in general form as

$$
\boldsymbol{R}_{g}^{\min }=\mathcal{R}_{h_{g}}^{\min } \otimes \mathcal{R}_{T X} \otimes \mathcal{R}_{R X},
$$

and consequently [23],

$$
\operatorname{rank}\left(\boldsymbol{R}_{g}^{\min }\right)=\operatorname{rank}\left(\mathcal{R}_{h_{g}}^{\min }\right) \operatorname{rank}\left(\mathcal{R}_{T X}\right) \operatorname{rank}\left(\mathcal{R}_{R X}\right) .
$$

Except for pathological setups exhibiting full spatial correlation between pairs of transmit or receive antennas (scenario not considered in this analysis), $\boldsymbol{\mathcal { R }}_{T X}$ and $\boldsymbol{\mathcal { R }}_{R X}$ are full rank matrices with $\operatorname{rank}\left(\boldsymbol{\mathcal { R }}_{T X}\right)=N_{T}$ and $\operatorname{rank}\left(\mathcal{R}_{R X}\right)=N_{R}$, and therefore,

$$
\operatorname{rank}\left(\boldsymbol{R}_{g}^{\min }\right)=N_{T} N_{R} \operatorname{rank}\left(\mathcal{R}_{h_{g}}^{\min }\right) .
$$

Therefore, the maximum attainable frequency diversity order can be directly related to $\boldsymbol{\mathcal { R }}_{h_{g}}^{\min }$ and is given by the number of independent paths in the channel delay profile. If error performance is to be optimized, enough subcarriers per group need to be allocated to ensure that $\operatorname{rank}\left(\mathcal{R}_{h_{g}}^{\min }\right)=P$. In fact, defining the sampled channel order $L$ as the channel delay spread in terms of chip (sampling) periods, it is shown in [5] that the maximum rank of $\mathcal{R}_{h_{g}}^{\min }$ is attained by setting the number of subcarriers per group to $Q=L+1$. While this is a valuable design rule in channels with short delay spread, in most practical scenarios where $L$ can be in the order of tens or even hundreds of samples, the theoretical number of subcarriers required to achieve full diversity would make the use of ML detection difficult even when using efficient search strategies (i.e., sphere decoding). Moreover, very often maximum diversity would only be attained at unreasonably large $E_{s} / N_{0}$ levels.

Since, for most realistic scenarios, setting the group size to guarantee full diversity $(Q=L+1)$ is unfeasible, we need to be able to measure what each additional subcarrier is contributing in terms of frequency diversity gain. Ideally, each additional subcarrier should bring along an extra diversity order, that is, an increase in $\operatorname{rank}\left(\mathcal{R}_{h_{g}}^{\min }\right)$ by one as it is indeed the case for uncorrelated channels. For correlated channels, however, this is often not the case and therefore to choose the group size it is useful to have some form of measure. A widely used tool in principal component analysis [24] to assess the practical dimensionality of a correlation matrix is the cumulative sum of eigenvalues (CSE) that, for the correlation matrix $\boldsymbol{\mathcal { R }}_{h_{g}}^{\min }$ with eigenvalues $\left\{\lambda_{h_{g}, q}\right\}_{q=1}^{Q}$, is defined as

$$
\Psi(n)=\frac{\sum_{q=1}^{n} \lambda_{h_{g}, q}}{\sum_{q=1}^{Q} \lambda_{h_{g}, q}} .
$$

For the frequency domain iid channel, $\Psi(n)$ is always a discrete linearly increasing function of $n$, and it can serve as a reference against which to measure the contribution of each subcarrier in arbitrary realistic channels. Based on the CSE concept in (48), a more meaningful and practical definition of rank $\left(\mathcal{R}_{h_{g}}^{\min }\right)$ is given as

$$
Q_{\epsilon}=\min \{n: \Psi(n) \geq 1-\epsilon\} \text {, }
$$


where $n \in\{1, \ldots, L+1\}$ and $\epsilon$ is a small non-negative value used to set a threshold on the CSE. Notice that $Q_{\epsilon} \rightarrow Q$ as $\epsilon \rightarrow 0$. Moreover, experiments with practical channel profiles show that, typically, $Q_{\epsilon}<<L+1$.

In conclusion, provided that scenarios with full spatial correlation are avoided, setting the number of subcarriers per group to $Q \leq Q_{\epsilon}$ yields

$$
\operatorname{rank}\left(\boldsymbol{R}_{g}^{\min }\right)=Q N_{T} N_{R}
$$

\subsubsection{On the rank of $G_{g, c}^{\min }$}

Given an $m \times n$ matrix $\boldsymbol{A}$ and an $n \times p$ matrix $\boldsymbol{B}$, it holds that [25]

$$
\begin{aligned}
\operatorname{rank}(\boldsymbol{A}) & +\operatorname{rank}(\boldsymbol{B})-n \leq \operatorname{rank}(\boldsymbol{A B}) \\
& \leq \min \{\operatorname{rank}(\boldsymbol{A}), \operatorname{rank}(\boldsymbol{B})\}
\end{aligned}
$$

Thus, using optimally rotated spreading codes and setting the number of subcarriers per group $Q$ using the proposed CSE-based approach, provided that pathological scenarios with full spatial correlation are avoided, we can use (44) and (50) in (51) to show that the global diversity order for the analysed MIMO strategies is given by

$$
\tilde{D}_{\min }=\operatorname{rank}\left(\boldsymbol{G}_{g, c}^{\min }\right)= \begin{cases}Q N_{R} & \mathrm{SDM} \\ Q N_{T} N_{R} & \mathrm{STBC} / \mathrm{CDD}\end{cases}
$$

\section{Reconfiguration strategies}

It is clear from (52) that the (instantaneous) rank of the group frequency channel correlation matrix $\mathcal{R}_{h_{g}}^{\text {min }}$ determines the asymptotic diversity of a MIMO-GO-CDM system, and therefore, it can form the basis for a group size adaptation mechanism. Strictly speaking, the maximum possible rank of $\mathcal{R}_{h_{g}}^{\mathrm{min}}$ is given by the number of independent paths in the channel profile. However, as shown in Subsection 3.3, very often the practical rank is far below this number as maximum diversity is only achieved at unrealistically low error rates. The adaptive group dimensioning scheme proposed next exploits this rank dependence to dynamically set the group size as a function of the channel response between all pairs of transmit and receive antennas. Figure 3 illustrates the architecture of the adaptive MIMO-GO-CDM system, where it can be appreciated that, in light of the acquired channel state information (CSI) and system constraints (complexity, QoS), the receiver determines the most appropriate group size to use and communicates this decision to the transmitter using a feedback channel. Note, as shown in Figure 3, that CSI and SNR information can also be used to determine the most appropriate modulation and coding scheme in conjunction with the GO-CDM dimensioning and MIMO mode selection. However this topic is beyond the scope of this study and in this work only fixed modulation and uncoded transmission modes are considered.

In order to perform the adaptive dimensioning of the GO-CDM component, the receiver requires an estimate $\tilde{\mathcal{R}}_{h_{g}}^{\text {min }}$ of the group frequency channel correlation matrix. An accurate estimate of the full correlation matrix $\mathcal{R}_{h_{g}}^{\min }$ could be computed by means of time averaging over the frequency domain, however, in indoor/WLANs scenarios where channels tend to vary very slowly, this approach would require of many OFDM symbols to get an adequate estimate. Fortunately, only the group channel correlation matrix is required, thus simplifying the correlation estimation. Exploiting the grouping structure of GO-CDM-MIMO-OFDM and assuming the channel frequency response is a wide-sense stationary (WSS) process, it is possible to derive an accurate estimate $\tilde{\mathcal{R}}_{h_{g}}^{\text {min }}$ from the instantaneous CSI, provided the subcarriers in a given group have been rightly chosen across the available bandwidth (see Section 2.2). It is assumed that the group size to be determined is chosen from a finite set of possible values $Q=\left\{Q^{1}, \ldots, Q^{\max }\right\}$ whose maximum, $Q^{\max }$, is limited by the maximum detection complexity the receiver can support. Suppose that at block symbol $k$ the receiver acquires knowledge of the channel to form the frequency response $\overline{\boldsymbol{h}}^{i j}(k)$ over all $N_{c}$ subcarriers. Now, using the maximum group size available, $Q^{\max }$, it is possible to form the frequency responses for all $N_{g}^{\min }=N_{c} / Q^{\max }$ groups, $\left\{\overline{\boldsymbol{h}}_{1}^{i j}(k), \ldots, \overline{\boldsymbol{h}}_{N_{g}^{i j}}^{i j}(k)\right\}$. Taking into account the WSS property it should hold that

$$
E\left\{\bar{h}_{g, q}^{i j}(k) \bar{h}_{g, v}^{i j}(k)\right\}=E\left\{\bar{h}_{m, q}^{i^{\prime} j^{\prime}}(k) \bar{h}_{m, v}^{i^{\prime} j^{\prime}}(k)\right\}
$$

for all pairs of transmit and receive antennas $(i, j)$ and $\left(i^{\prime}, j^{\prime}\right)$ and any $q, v \in\left\{1, \ldots, Q^{\max }\right\}$, as the correlation among any two subcarriers should only depend on their separation, not their absolute position or the transmit/receive antenna pair. A group channel correlation matrix estimate from a single frequency response can now be formed averaging across transmit and receive antennas, and groups,

$$
\tilde{\boldsymbol{R}}_{h_{g}}^{\min }=\frac{1}{N_{T} N_{R} N_{g}^{\min }} \sum_{i=1}^{N_{T}} \sum_{j=1}^{N_{R}} \sum_{g=1}^{N_{g}^{\min }} \overline{\boldsymbol{h}}_{g}^{i j}(k)\left(\overline{\boldsymbol{h}}_{g}^{i j}(k)\right)^{H}
$$

Using basic properties regarding the rank of a matrix, it is easy to prove that $\operatorname{rank}\left(\tilde{\mathcal{R}}_{h_{g}}^{\min }\right) \leq \min \left(N_{g}^{\min }, Q^{\max }\right)$, therefore, $N_{g}^{\min }=Q^{\max }$ maximises the range of possible group sizes using a single CSI shot. Let us denote the non-increasingly ordered positive eigenvalues of $\tilde{\mathcal{R}}_{h_{g}}^{\min }$ by 


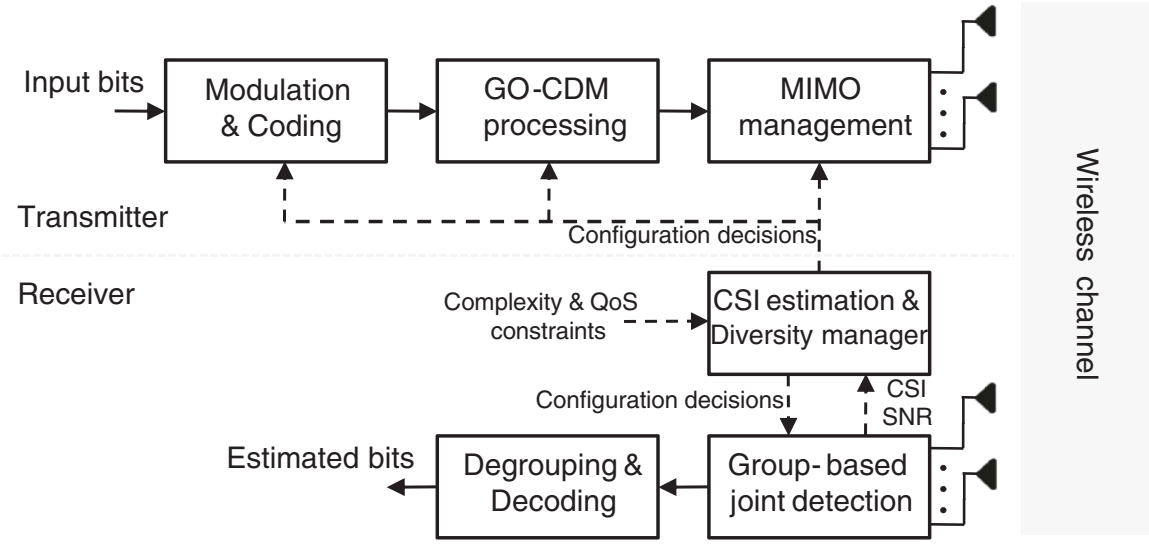

Figure 3 Communication architecture for a MIMO-GO-CDM with group-size adaptation.

$\tilde{\Lambda}_{h_{g}}=\left\{\tilde{\lambda}_{h_{g}, q}\right\}_{q=1}^{\tilde{Q}}$ where, owing to the deterministic character of $\tilde{\mathcal{R}}_{h_{g}}^{\min }$, they can all be assumed to be different and with order one, and consequently, $\tilde{Q}$ represents the true rank of $\tilde{\mathcal{R}}_{h_{g}}^{\text {min }}$. For the purpose of on-line adaptation, an analogous of the CSE-based definition of rank in (48) can now be given based on estimated (rather than true) eigenvalues,

$$
\tilde{Q}_{\epsilon}=\min \left\{n: \Psi(n)=\frac{\sum_{q=1}^{n} \tilde{\lambda}_{h_{g}, q}}{\sum_{q=1}^{\tilde{Q}} \tilde{\lambda}_{h_{g}, q}} \geq 1-\epsilon\right\} .
$$

Since the group size $Q$ represents the dimensions of an orthonormal spreading matrix $\boldsymbol{C}$, restrictions apply on the range of values it can take. For instance, in the case of (rotated) Walsh-Hadamard matrices, $Q$ is constrained to be a power of two. The mapping of $\tilde{Q}_{\epsilon}$ to an allowed group dimension, jointly with the setting of $\epsilon$, permits the implementation of different reconfiguration strategies, e.g.,

$$
\begin{aligned}
& \text { Maximise performance : } Q=\underset{\hat{Q} \in Q}{\arg \min }\left\{\hat{Q} \geq \tilde{Q}_{\epsilon}\right\} \\
& \text { Minimise complexity : } Q=\underset{\hat{Q} \in Q}{\arg \min }\left\{\left|\hat{Q}-\tilde{Q}_{\epsilon}\right|\right\}
\end{aligned}
$$

It is difficult to assess the feedback involved in this adaptive diversity mechanism as it depends on the dynamics of the underlying channel. The suggested strategy to implement this procedure is that the receiver regularly estimates the group channel rank and whenever a variation occurs, it determines and feeds back the new group dimension to the transmitter. In any case, the feedback information can be deemed insignificant as every update just requires of $\left\lceil\log _{2} \mathcal{Q}\right\rceil$ feedback bits with $\mathcal{Q}$ denoting the cardinality of set $\boldsymbol{Q}$. Differential encoding of $\mathcal{Q}$ would bring this figure further down.

\section{Computational complexity considerations}

The main advantage of the group size adaptation technique introduced in the previous section is a reduction of computational complexity without any significant performance degradation. To gain some further insight, it is useful to consider the complexity of the detection process taking into account the group size in the GO-CDM component while assuming that an efficient ML implementation, such as the one introduced in [17], is in use. To this end, [26] demonstrated that the number of expected (complex) operations in an efficient ML detector operating at reasonable SNR levels is roughly cubic with the number of symbols jointly detected. That is, to detect one single group in a MIMO-GO-CDM system, $\Omega_{g}=\mathcal{O}\left(N_{Q}^{3}\right)$ operations are required.

Obviously, to detect all groups in the system, the expected number of required operations is given by $\Omega_{T}=$ $\frac{N_{c}}{Q} \Omega_{g}$. Figure 4 depicts the expected per-group and total complexity for a system using $N_{c}=64$ subcarriers, a set of possible group sizes given by $\{1,2,4,8\}$ and different number of transmitted streams. Note that, in the context of this paper, $N_{s}>1$ necessarily implies the use of SDM. Importantly, increasing the group size from $Q=1$ to $Q=8$ implies an increase in the number of expected operations of more than two orders of magnitude, thus reinforcing the importance of rightly selecting the group size to avoid a huge waste in computational/power resources. Finally, it should be mentioned that for the STBC setup, efficient detection strategies exist that decouple the Alamouti decoding and GO-CDM detection resulting in a simplified receiver architecture that is still optimum $[27,28]$. 


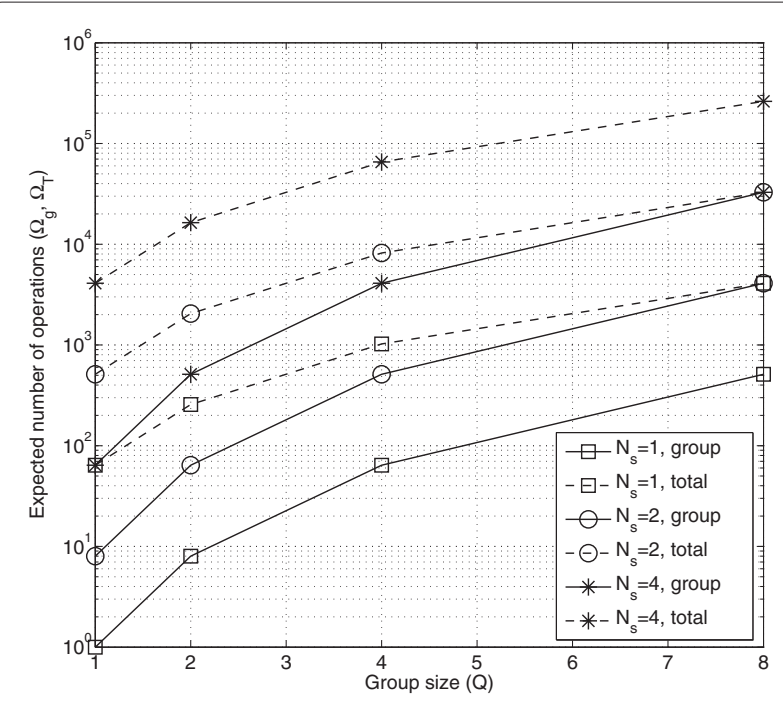

Figure 4 Complexity as a function of group size (Q) for different number of transmitted streams.

\section{Numerical Results}

In this section, numerical results are presented with the objective of validating the analytical derivations introduced in previous sections and also to highlight the benefits of the adaptive MIMO-GO-CDM architecture. The system considered employs $N_{c}=64$ subcarriers within a $B=20 \mathrm{MHz}$ bandwidth. These parameters are representative of modern WLAN systems such as IEEE 802.11n [29]. The GO-CDM technique has been applied by spreading the symbols forming a group with a rotated Walsh-Hadamard matrix of appropriate size. The set of considered group sizes is given by $Q=\{1,2,4,8\}$. This set covers the whole range of practical diversity orders for WLAN scenarios while remaining computationally feasible at reception. Note that a system with $Q=1$ effectively disables the GO-CDM component. For most of the results shown next, Channel Profile E from [30] has been used. Perfect channel knowledge is assumed at the receiver. Regarding the MIMO aspects, the system is configured with two transmit and two receive antennas $\left(N_{T}=\right.$ $\left.N_{R}=2\right)$. As in [16], the correlation coefficient between $\mathrm{Tx}(\mathrm{Rx})$ antennas is defined by a single coefficient $\rho_{T x}$ $\left(\rho_{R x}\right)$. Note that in order to make a fair comparison among the different spatial configurations, different modulation alphabets are used. For SDM, two streams are transmitted using BPSK whereas for STBC and CDD, a single stream is sent using QPSK modulation, ensuring that the three configurations achieve the same spectral efficiency.

Figure 5 presents results for SDM, CDD and STBC when transmit and receive correlation are set to $\rho_{T x}=0.25$ and $\rho_{R x}=0.75$, respectively. The first point to highlight from the three subfigures is the excellent agreement between simulated and analytical results for the usually relevant range of BERs $\left(10^{-3}-10^{-7}\right)$. It can also be observed the various degrees of influence exerted by the GO-CDM component depending on the particular spatial processing mechanism in use. For example, at a $P_{b}=10^{-4}$, it can be observed that in SDM and CDD, the maximum group size considered $(Q=8)$ brings along SNR reductions greater than $10 \mathrm{~dB}$ when compared to the setup without GO-CDM $(Q=1)$. In contrast, in combination with STBC, the maximum gain offered by GO-CDM is just above 5 $\mathrm{dB}$. The overall superior performance of STBC can be explained by the fact that it exploits transmit and receive diversity whereas in SDM there is no transmit diversity and in CDD, this is only exploited when combined with GO-CDM and/or channel coding.
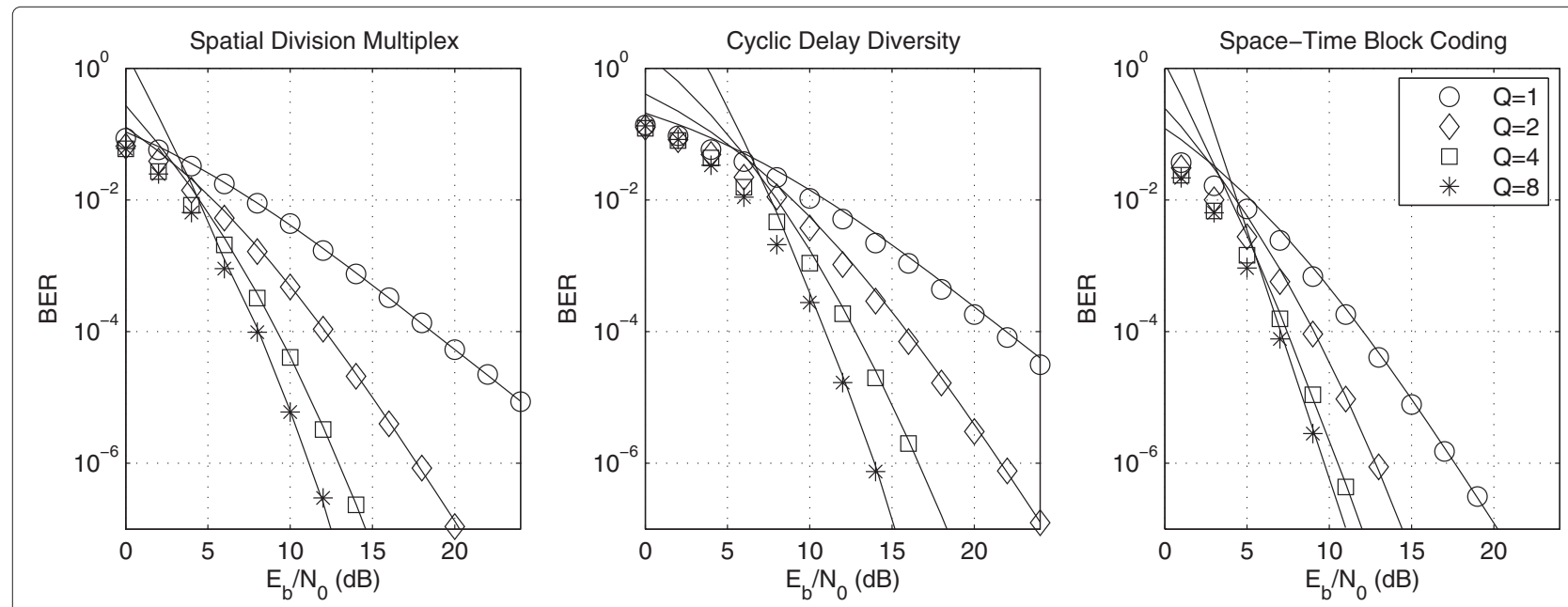

Figure 5 Analytical (lines) and simulated (markers) BER for GO-CDM configured to operate in SDM (left), CDD (centre) and STBC (right) for different group sizes in Channel Profile E. 


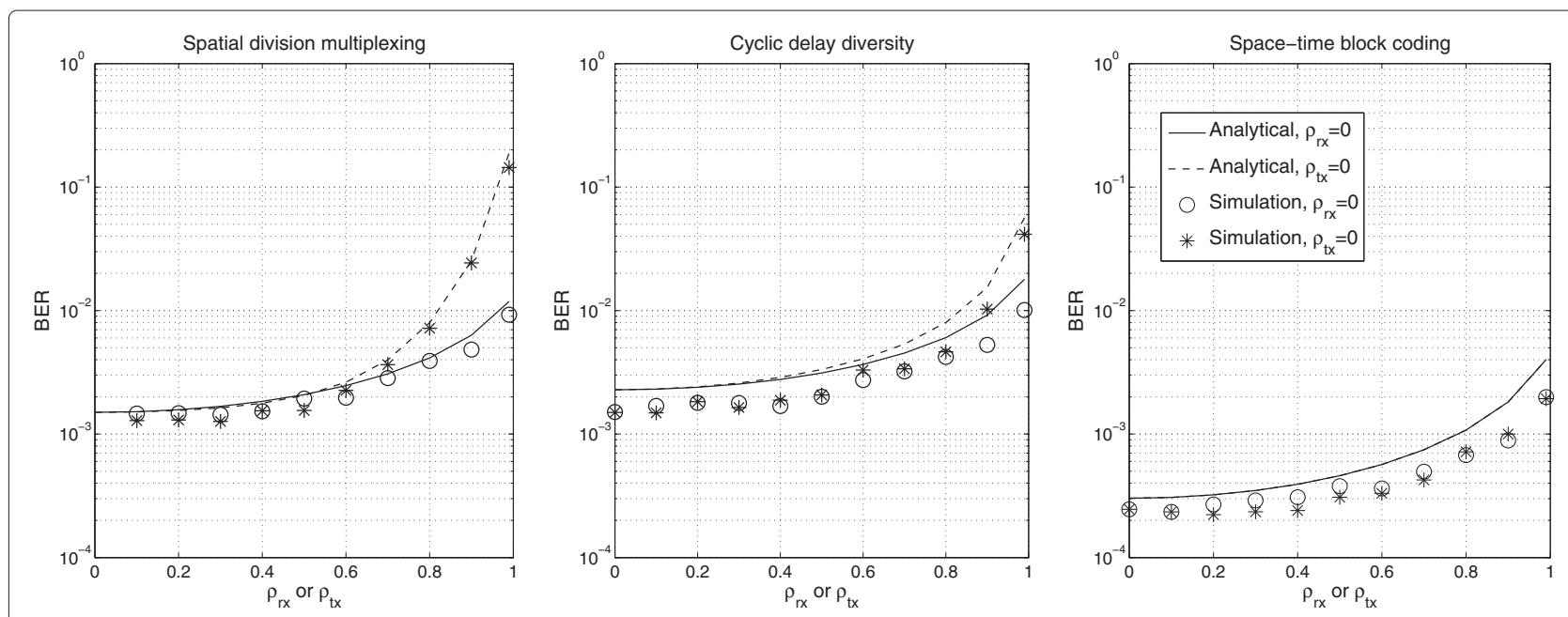

Figure 6 Analytical (lines) and simulated (markers) BER for GO-CDM configured to operate in SDM (left), CDD (centre) and STBC (right) for different transmit/antenna correlation values.

Next, the effects of antenna correlation at either side of the communication link have been assessed for each of the MIMO processing schemes. To this end, the MIMOGO-CDM system has been configured with $Q=2$ and the SNR fixed to $E_{s} / N_{0}=10 \mathrm{~dB}$. The antenna correlation at one side has been set to 0 when varying the antenna correlation at the other end between 0 and 0.99 . As seen in Figure 6, a good agreement between analytical and numerical results can be appreciated. The small discrepancy between theory and simulation is mainly due to the use of the union bound, which always overestimates the true error rate. In any case, the theoretical expressions are able to predict the performance degradation due to an increased antenna correlation. Note that, in CDD and $\mathrm{SDM}$, for low to moderate values $(0.0-0.7)$, correlation at either end results in a similar BER degradation, however, for large values $(>0.7)$, correlation at the transmitter is significantly more deleterious than at the receiver. For the STBC scenario, analysis and simulation demonstrate that it does not matter which communication end suffers from antenna correlation as it leads to exactly the same results. This is because all symbols are transmitted and received through all antennas ( $\mathrm{Tx}$ and $\mathrm{Rx}$ ) and therefore equally affected by the correlation at both ends.

Finally, the performance of the proposed group adaptive mechanism has been assessed by simulation. The SNR
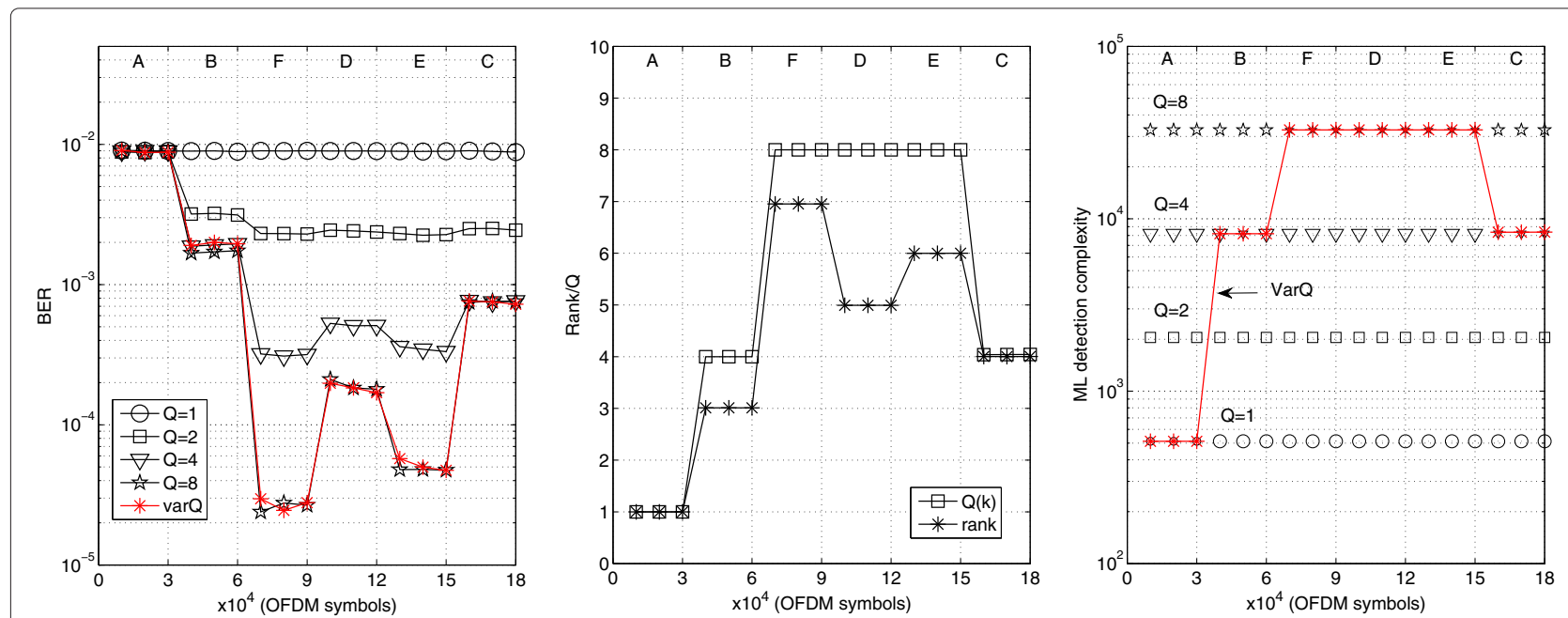

Figure 7 Behaviour of fixed and adaptive MIMO GO-CDM-OFDM over varying channel profile using QPSK modulation at $E_{s} / N_{0}=12 \mathrm{~dB}$. $N_{T}=N_{R}=N_{S}=2$ (SDM mode). Left: epoch-averaged BER performance. Middle: epoch-averaged rank/group size. Right: epoch-averaged detection complexity. 
has been fixed to $E_{s} / N_{0}=12 d B$ and a time varying channel profile has been generated. This profile is composed of epochs of 10,000 OFDM symbols each. Within an epoch, an independent channel realisation for each OFDM symbol is drawn (quasi-static block fading) from the same channel profile. For visualisation clarity, the generating channel profile is kept constant for three consecutive epochs and then it changes to a different one. All channel profiles (A-F) from IEEE 802.11n [30] have been considered. Results shown correspond to an SDM configuration.

The left plot in Figure 7 shows the BER evolution for fixed and adaptive group size systems as the environment switches among the different channel profiles. The uppercase letter on the top of each plot identifies the particular channel profile for a given epoch. Each marker represents the averaged BER of 10,000 OFDM symbols. Focusing on the fixed group configurations it is easy to observe that a large group size does not always bring along a reduction in BER. For example, for Profile A (frequency-flat channel) there is no benefit in pursuing extra frequency diversity at all. Similarly, for Profiles B and C there is no advantage in setting the group size to values larger than 4 . This is in fact the motivation of the proposed MIMO adaptive group size algorithm denoted in the figure by varQ. It is clear from the middle plot in Figure 7 that the proposed algorithm is able to adjust the group size taking into account the operating environment so that when the channel is not very frequency selective low $Q$ values are used and, in contrast, when large frequency selectivity is sensed the group size dimension grows.

Complementing the BER behaviour, it is important to consider the computational cost of the configurations under study. To this end the right plot in Figure 7 shows the expected number of complex operations (see Section 5). In this plot it can be noticed the huge computational waste incurred, since there is no BER reduction, in the fixed group size systems with large $Q$ when operating in channels with a modest amount of frequencyselectivity (A, B and C).

\section{Conclusions}

This paper has introduced the combination of GO-CDM and multiple transmit antenna technology as a means to simultaneously exploit frequency, time and space diversity. In particular, the three most common MIMO mechanisms, namely, SDM, STBC and CDD, have been considered. An analytical framework to derive the BER performance of MIMO-GO-CDM has been presented that is general enough to incorporate transmit and receive antenna correlations as well as arbitrary channel power delay profiles. Asymptotic results have highlighted which are the important parameters that influence the practical diversity order the system can achieve when exploiting the three diversity dimensions. In particular, the channel correlation matrix and its effective rank, defined as the number of significant positive eigenvalues, have been shown to be the key elements on which to rely when dimensioning MIMO-GO-CDM systems. Based on this effective rank, a dynamic group size strategy has been introduced able to adjust the frequency diversity component (GO-CDM) in light of the sensed environment. This adaptive MIMO-GO-CDM has been shown to lead to important power/complexity reductions without compromising performance and it has the potential to incorporate other QoS requirements (e.g., delay, target BER) that may result in further energy savings. Simulation results using IEEE $802.11 \mathrm{n}$ parameters have served to verify three facts. Firstly, MIMO-GO-CDM is a versatile architecture to exploit the different degrees of freedom the environment has to offer. Secondly, the presented analytical framework is able to accurately model the BER behaviour of the various MIMO-GO-CDM configurations. Lastly, the adaptive group size strategy is able to recognize the operating environment and adapt the system appropriately. Future efforts will focus on extending the analytical results presented here to setups including error correction techniques.

\section{Competing interests}

The authors declare that they have no competing interests.

\section{Acknowledgements}

This work has been supported in part by MEC and FEDER under projects COSMOS (TEC2008-02422) and AM3DIO (TEC2011-25446), Spain.

This work has been supported in part by MICINN, MINECO and FEDER under projects COSMOS (TEC2008-02422) and AM3DIO (TEC2011-25446), Spain.

Received: 2 May 2011 Accepted: 20 July 2012

Published: 20 July 2012

\section{References}

1. G Stuber, J Barry, S Mclaughlin, Y Li, M Ingram, T Pratt, Broadband MIMO-OFDM wireless communications. Proc. IEEE. 92(2), 271-294 (2004)

2. S Kaiser, OFDM code-division multiplexing in fading channels. IEEE Trans. Commun. 50, 1266-1273 (2002)

3. N Yee, J-P Linnartz, G Fettweis, Multi-carrier CDMA in indoor wireless radio networks. in Proc. IEEE Int. Symp. on Pers., Indoor and Mob. Rad. Comm, (Yokohama, Japan, Sept 1993), pp. 109-113

4. F Riera-Palou, G Femenias, J Ramis, On the design of uplink and downlink group-orthogonal multicarrier wireless systems. IEEE Trans. Commun. 56(10), 1656-1665 (2008)

5. X Cai, S Zhou, G Giannakis, Group-orthogonal multicarrier CDMA. IEEE Trans. Commun. 52(1), 90-99 (2004)

6. F Riera-Palou, G Femenias, OFDM with adaptive frequency diversity. IEEE Signal Process. Lett. 16(10), 837-840 (2009)

7. E Telatar, Capacity of multi-antenna Gaussian channels. Eur. Trans. Telecommun. 10(6), 585-595 (1999)

8. G Foschini, Layered space-time architecture for wireless communication in a fading environment when using multi-element antennas. Bell Labs Tech. J. 1(2), 41-59 (1996)

9. A Alamouti, A simple transmit diversity technique for wireless communications. IEEE JSAC. 16, 1451-1458 (1998)

10. V Tarokh, $\mathrm{H}$ Jafarkhani, A Calderbank, Space-time block codes from orthogonal designs. IEEE Trans. Inf. Theory. 45(5), 1456-1467 (1999) 
11. S Wittneben, A new bandwidth efficient transmit antenna modulation diversity scheme for linear digital modulation. in Proc. IEEE Int. Conf. on Commun, (Geneva, Switzerland, May 1993), pp. 1630-1634

12. A Lodhi, F Said, M Dohler, A Aghvami, Performance comparison of space-time block coded and cyclic delay diversity mc-cdma systems. IEEE Wirel. Commun. 12(2), 38-45 (2005)

13. R Heath, A Paulraj, Switching between diversity and multiplexing in MIMO systems. IEEE Trans. Commun. 53(6), 962-968 (2005)

14. F Riera-Palou, G Femenias, An efficient adaptive frequency diversity scheme for IEEE $802.11 \mathrm{n}$ networks. in Proc. IEEE Global Telecommunications, Conference (Globecom 2009), (Honolulu, US, Dec 2009)

15. G Bauch, J Malik, Cyclic delay diversity with bit-interleaved coded modulation in orthogonal frequency division multiple access. IEEE Trans. Wirel. Commun. 8, 2092-2100 (2006)

16. A van Zelst, J Hammerschmidt, A single coefficient spatial correlation model for multiple-input multiple-output (mimo) radio channels. in Proc. Proc. URSI XXVIIth General Assembly, (Maastricht, The Netherlands, Aug 2002), pp. 1-4

17. U Fincke, M Pohst, Improved methods for calculating vectors of short length in a lattice, including a complexity analysis. Math. Comput. 44, 463-471 (1985)

18. M Simon, S Hinedi, W Lindsey, Digital communication techniques: Signal design and detection. (Englewood Cliffs, Prentice Hall PTR, 1995)

19. JW Craig, A new, simple and exact result for calculating the probability of error for two-dimensional signal constellations. in IEEE MILCOM'91 Conf. Rec, (Boston, MA, 1991), pp. 25.5.1-25.5.5

20. G Femenias, BER performance of linear STBC from orthogonal designs over MIMO correlated Nakagami-m fading channels. IEEE Trans. Veh. Technol. 53(2), 307-317 (2004)

21. S Amari, R Misra, Closed-form expressions for distribution of sum of exponential random variables. IEEE Trans. Reliab. 46(4), 519-522 (1997)

22. A Bury, J Egle, J Lindner, Diversity comparison of spreading transforms for multicarrier spread spectrum transmission. IEEE Trans. Commun. 51(5), 774-781 (2003)

23. KB Petersen, MS Pedersen, The matrix cookbook, Oct 2008, version 20081110. [Online]. Available: http://www2.imm.dtu.dk/pubdb/p.php? 3274

24. R Johnson, D Wichern, Applied Multivariate Statistical Analysis, 5th ed. (Upper Saddle River, Prentice Hall, 2002)

25. C Meyer, Matrix analysis and applied linear algebra. (Philadelphia, Society for Industrial and Applied Mathematics (SIAM), 2000)

26. H Vikalo, B Hassibi, On the sphere-decoding algorithm ii. generalizations, second-order statistics, and applications to communications. IEEE Trans. Signal Process. 53(8), 2819-2834 (2005)

27. F Riera-Palou, G Femenias, Improving STBC performance in IEEE $802.11 \mathrm{n}$ using group-orthogonal frequency diversity. in Proc. IEEE Wireless Communications and, Networking Conference, (Las Vegas, US, Apr 2008), pp. 1-6

28. F Riera-Palou, G Femenias, Group-orthogonal code-division multiplex: a physical-layer enhancement for IEEE $802.11 \mathrm{n}$ networks. EURASIP J. Wirel. Commun. Netw. 2010, 1-10 (2010)

29. IEEE, Part 11: Wireless LAN Medium Access Control (MAC) and Physical Layer (PHY) Specifications Amendment 5: Enhancements for Higher Throughput. IEEE Std 802.11n-2009, 2009

30. V Erceg, Indoor MIMO WLAN Channel Models. doc.: IEEE 802.11-03/871ro, Draft proposal, Nov 2003

doi:10.1186/1687-1499-2012-226

Cite this article as: Riera-Palou and Femenias: A unified view of diversity in multiantenna-multicarrier systems: analysis and adaptation strategies. EURASIP Journal on Wireless Communications and Networking 2012 2012:226. 